\title{
Advanced Applications of Neural Networks and Artificial Intelligence: A Review
}

\author{
Koushal Kumar \\ Department of CSE/IT, Lovely Professional University, Phagwara, Punjab India \\ kaushal_kumar302@yahoo.com \\ Gour Sundar Mitra Thakur \\ Department of CSE/IT, Lovely Professional University, Phagwara, Punjab, India \\ cse.gsmt@gmail.com
}

\begin{abstract}
Artificial Neural Network is a branch of Artificial intelligence and has been accepted as a new computing technology in computer science fields. This paper reviews the field of Artificial intelligence and focusing on recent applications which uses Artificial Neural Networks (ANN's) and Artificial Intelligence (AI). It also considers the integration of neural networks with other computing methods Such as fuzzy logic to enhance the interpretation ability of data. Artificial Neural Networks is considers as major soft-computing technology and have been extensively studied and applied during the last two decades. The most general applications where neural networks are most widely used for problem solving are in pattern recognition, data analysis, control and clustering. Artificial Neural Networks have abundant features including high processing speeds and the ability to learn the solution to a problem from a set of examples. The main aim of this paper is to explore the recent applications of Neural Networks and Artificial Intelligence and provides an overview of the field, where the AI \& ANN's are used and discusses the critical role of AI \& NN played in different areas.
\end{abstract}

Index Terms - Artificial intelligence, Neural Networks, Machine learning, Soft computing

\section{Introduction To Artificial Intelligence}

$$
\text { Intelligence }=\text { perceive }+ \text { Analyse }+ \text { React }
$$

Artificial intelligence (AI) is defined as intelligence exhibited by an artificial entity to solve complex problems and such a system is generally assumed to be a computer or machine [1]. Artificial Intelligence is an integration of computer science and physiology Intelligence in simple language is the computational part of the ability to achieve goals in the world. Intelligence is the ability to think to imagine creating memorizing and understanding, recognizing patterns, making choices adapting to change and learn from experience. Artificial intelligence concerned with making computers behave like humans. AI tries to solve the complex problems in more human like fashion and in much less time then a human takes. Hence it is called as Artificial Intelligence [2]. Artificial intelligence can be divided into parts according to philosophy of AI.

a) Strong AI

b) Weak AI

\section{What is Strong AI?}

The principle behind Strong AI is that the machines could be made to think or in other words could represent human minds in the future. Thus Strong AI claims that in near future we will be surrounded by such kinds of machine which can completely works like human being and machine could have human level intelligence. If that is the case, those machines will have the ability to reason, think and do all functions that a human is capable of doing. Current research is nowhere near creating strong AI, and a lively debate is ongoing as to whether this is even possible [3].

\section{What is weak AI?}

The principle behind Weak AI is simply the fact that machines can be made to act as if they are intelligent. Weak AI simply states that thinking like features can be easily added to computer to make them more useful tools and this already started to happen. For example, when a human player plays chess against a computer, the human player may feel as if the computer is actually making impressive moves. But the chess application is not thinking and planning at all. All the moves it makes are previously fed in to the computer by a human and that is how it is ensured that the software will make the right moves at the right times. More examples of Weak AI are witness expert systems, drive by wires cars and speech recognisation systems [4].

Turing Test: The Turing test is a test of a machine's ability to exhibit intelligent behavior. The test was introduced by Alan Turing in his 1950 paper Computing Machinery and Intelligence. The original question behind this test was "Can machines think? ". The test proceeds as follows a human judge engages in a natural language conversation with one human and one machine, each of which tries to appear human. All participants are 
placed in isolated locations. If the judge cannot reliably tell the machine from the human, the machine is said to have passed the test. In order to test the machine's intelligence rather than its ability to render words into audio, the conversation is limited to a text-only channel such as a computer keyboard and screen." Sufficiently many interrogators are unable to distinguish the computer from the human being then it is to be concluded that the computer thinks [5].

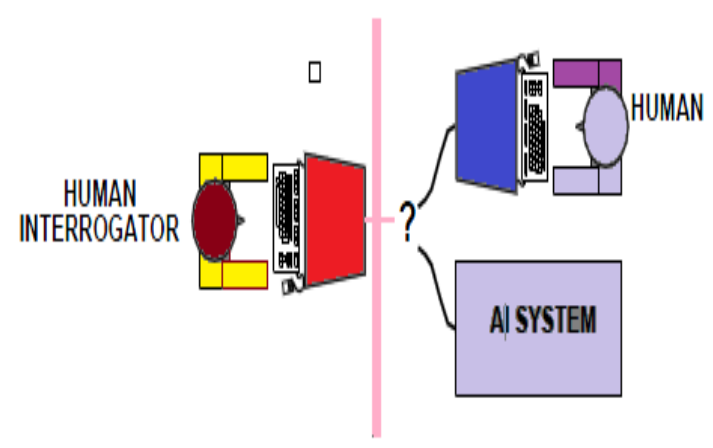

Fig 1 Turing test for checking machine intelligence

\section{Roots of AI}

Artificial Intelligence has identifiable roots in a number of older disciplines, particularly

- Philosophy

- Logic/Mathematics

- Computation

- Psychology/Cognitive Science

- Biology/Neuroscience

There is inevitably much overlap Example, between philosophy and logic, or between mathematics and computation. By looking at each of these in turn, we can gain a better understanding of their role in AI, and how these underlying disciplines have developed to play that role.

Goal of AI: An important goal of AI research is to devise machines to perform various tasks which normally require human intelligence

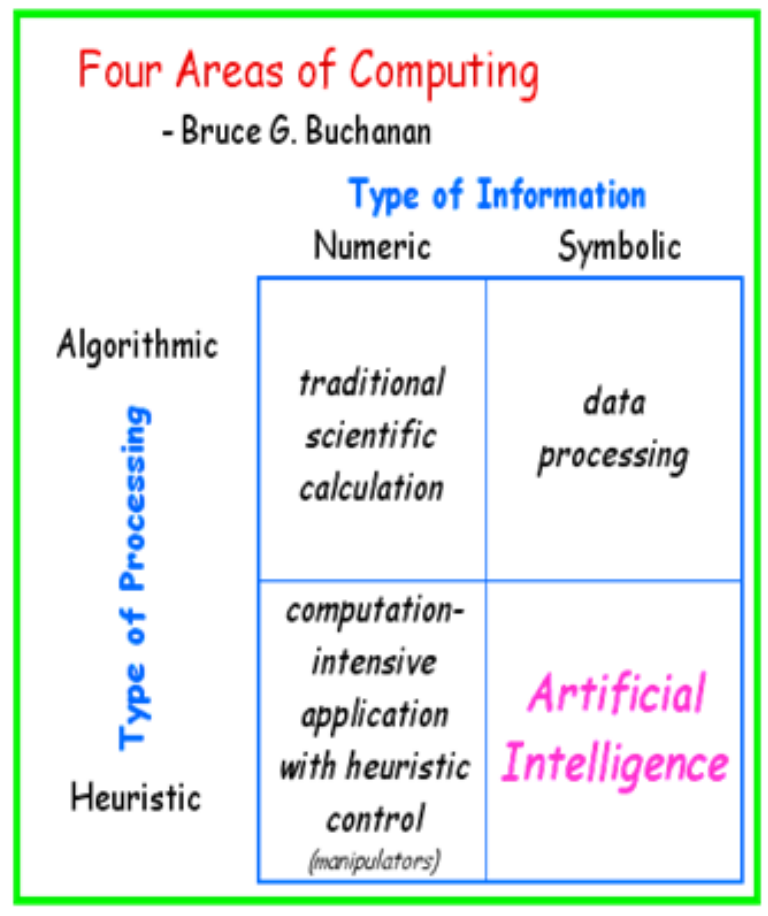

Fig. 2 Areas of computing

\section{Introduction To Artificial Neural Networks}

Artificial neural networks (ANN) have been developed as generalizations of mathematical models of biological nervous systems. A first wave of interest in neural networks emerged after the introduction of simplified neurons by McCulloch and Pitts (1943) also known as connectionist models. An Artificial Neural Network is a network of collections of very simple processors ("Neurons") each possibly having a (small amount of) local memory. The units operate only on their local data and on the inputs they receive via the connections or links which are unidirectional [6].A network unit has a rule for summing the signals coming in and a rule for calculating an output signal that is then, sent to other network units. According to Callen the rules for calculating the output is known as the activation function [7]. A neural network has three layers in its structure. First layer is input layer which is directly interact with external worlds; second layer is of hidden unit where computation is done according to function provided, the last layer is output layer from where we get output. Knowledge in neural networks is stored as synaptic weights between neurons. The network propagates the input data from layer to layer until the output data is generated. If the networks is multilayer perceptron with Backpropogation algorithm and the output is different from the desire output, then an error is calculated and propagated backwards through the network. The synaptic weights are modified as the error is propagated [8]. Now a Days Researchers are attempting to build a silicon-based electronic network that is modelled on the working and form of the human brain and this is shown in Fig 3. Generalization is the only ability that makes ANNs so powerful tool. How the human brain works, it learns to realize patterns and 
remembers them. Similarly, the neural networks developed have the ability to learn patterns and remember [9].

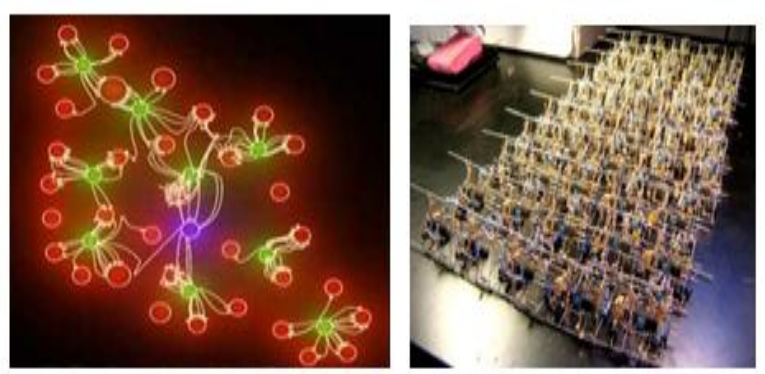

Fig. 3 Biological vs. Artificial Neural Networks
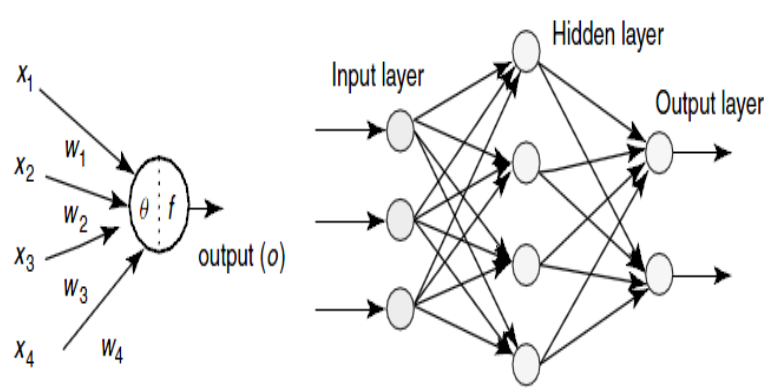

$\begin{array}{ll}\text { (a) Artificial neuron } & \text { (b)Multilayered artificial neural network }\end{array}$

Fig 4 Architecture of an artificial neuron and a multilayered neural network

The learning methods in neural networks can be classified into three categories. These are supervised learning, unsupervised learning and reinforcement learning

\section{A. Types Of Learning In Neural Networks}

Supervised Learning: In supervised learning networks inputs as well as corresponding output is given to the networks. In forward the errors or discrepancies between the desired and actual response for each node in the output layer are found. These are then used to determine weight changes in the net according to the prevailing learning rule. The term supervised originates from the fact that the desired signals on individual output nodes are provided by an external teacher. Examples of supervised learning are the delta rule, and the perceptron rule [10]

Unsupervised Learning: In this type of learning external teacher is not present. So this kind of learning is based upon clustering technique. According to inputs patterns clusters are divided into different classes. This kind of learning is also called self organization. Typical examples are the Hebbian learning rule and the competitive learning rule. Unsupervised learning is much more important then supervised learning since it likely to be much more common in the brain then supervised learning. The kind of learning is determined by the way in which the changes to network parameters have done.
Reinforcement learning: This kind of learning is based upon both supervised and unsupervised learning. Reinforcement learning is learning what to do how to map situations to actions so as to maximize a numerical reward signal. In this learning there is reward for correct outputs and penalty for wrong outputs. Reinforcement learning is also called learning with a critic as opposed to learning with a teacher [11]

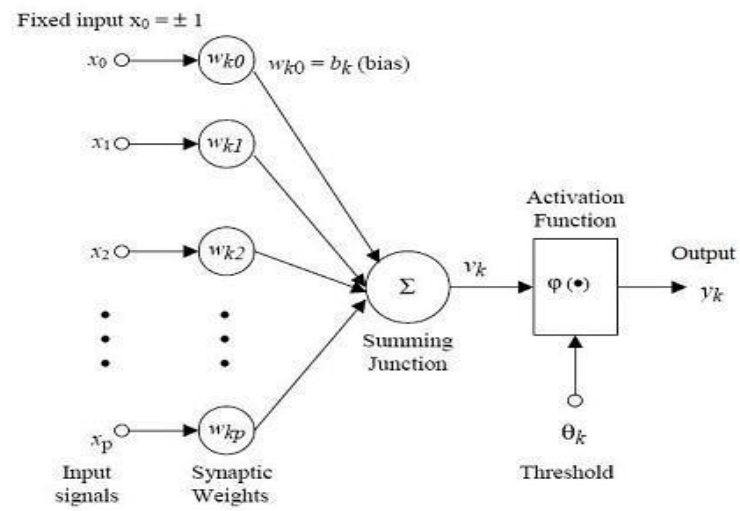

Fig 5 Mathematical model of neural network

In figure 3 wk0, wk1.....wkp are all are weights to determine the strengths of inputs vectors $\mathrm{Xn}$ vectors. Each input is multiplied with weight vectors.

$$
v_{k}=\sum_{j=1}^{p} w_{k j} x_{j}
$$

The output of the neuron yk is outcome of some activation function on the value of $\mathrm{vk}$.

\section{B. Activation Functions}

An activation function $\Phi($.$) performs a mathematical$ operation on the outputs of neuron $\mathrm{Vk}$. The activation functions are selected according to the types of problem to be solved by the network. In general, there are three types of activation functions, denoted by $\Phi$ (.) First, there is the Threshold Function which takes on a value of 0 if the summed input is less than a certain threshold value $(v)$, and the value 1 if the summed input is greater than or equal to the threshold value.

$$
\varphi(v)= \begin{cases}1 & \text { if } v \geq 0 \\ 0 & \text { if } v<0\end{cases}
$$

Secondly, there is the Piecewise-Linear function. This function again can take on the values of 0 or 1 , but can also take on values between that depending on the amplification factor in a certain region of linear operation. 


$$
\varphi(v)=\left\{\begin{array}{cc}
1 & v \geq \frac{1}{2} \\
v & -\frac{1}{2}>v>\frac{1}{2} \\
0 & v \leq-\frac{1}{2}
\end{array}\right.
$$

Thirdly, there is the sigmoid function. This function can range between 0 and 1 , but it is also sometimes useful to use the -1 to 1 range. An example of the sigmoid function is the hyperbolic tangent function [12].

$$
\varphi(v)=\tanh \left(\frac{v}{2}\right)=\frac{1-\exp (-v)}{1+\exp (-v)}
$$

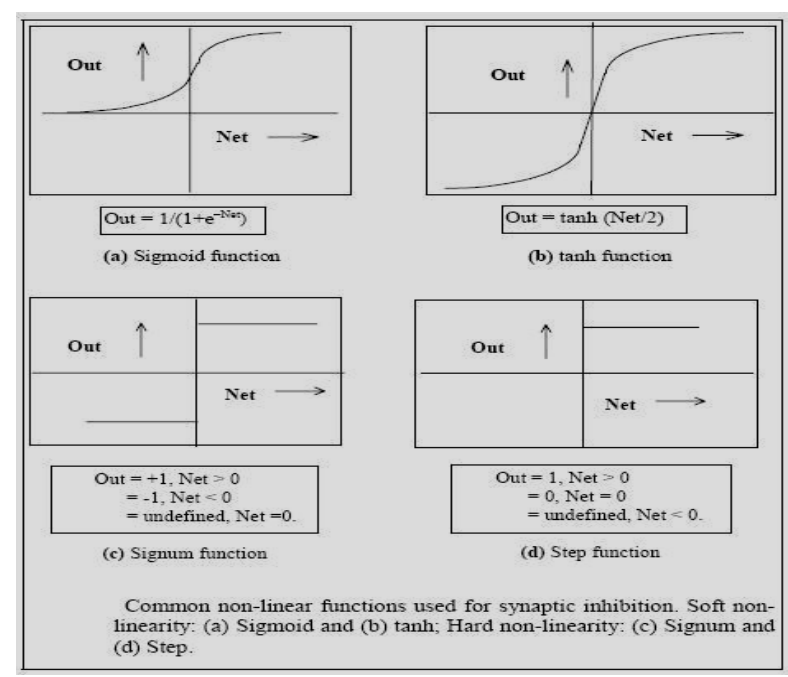

Fig 6 Different activations functions with activation curve

Table 1: A Brief History of The Fields

\begin{tabular}{|l|l|}
\hline Years & \multicolumn{1}{|c|}{ History } \\
\hline $\mathbf{1 9 4 3}$ & $\begin{array}{l}\text { McCulloch and Pitts proposed the } \\
\text { McCulloch pits neuron model }\end{array}$ \\
\hline $\mathbf{1 9 4 9}$ & Hebb proposed the Hebbian learning rule \\
\hline $\mathbf{1 9 5 8}$ & $\begin{array}{l}\text { Rosenblatt introduced the simple single layer } \\
\text { network called perceptron }\end{array}$ \\
\hline $\mathbf{1 9 6 9}$ & $\begin{array}{l}\text { Minskey and Papert's demonstrated the } \\
\text { limitation of single layer perceptron }\end{array}$ \\
\hline $\mathbf{1 9 8 2}$ & $\begin{array}{l}\text { Hopfield published number of papers on } \\
\text { Hopfield networks }\end{array}$ \\
\hline $\mathbf{1 9 8 2}$ & $\begin{array}{l}\text { Kohenon developed the self organizing maps } \\
\text { that now bear his name }\end{array}$ \\
\hline $\mathbf{1 9 8 6}$ & $\begin{array}{l}\text { The back propagation learning algorithm for } \\
\text { multilayer perceptron was developed }\end{array}$ \\
\hline $\mathbf{1 9 9 0}$ & $\begin{array}{l}\text { The sub-field of radial basis function } \\
\text { network was developed }\end{array}$ \\
\hline $\mathbf{2 0 0 0}$ & $\begin{array}{l}\text { The power of ensemble of neural networks } \\
\text { and support vector machines become } \\
\text { apparent }\end{array}$ \\
\hline
\end{tabular}

\section{Applications of Neural Networks}

Evolution of Neural Networks for Control of Pursuit \& Evasion: Researchers in the field of artificial life
(Alife) is frequently moved towards the domain of Pursuit \& Evasion to study the evolution of complex agent behaviors [13]. The MPEG movie sequences illustrate behaviors generated by dynamical recurrent neural network controllers co-evolved for pursuit and evasion capabilities is shown below in figure 2. From an initial population of random network designs, successful designs in each generation are selected for reproduction with recombination, mutation, and gene duplication. Selection is based on measures of how well each controller performs in a number of pursuit-evasion contests. In each contest a pursuer controller and an evader controller are pitched against each other, controlling simple "visually guided" 2-dimensional autonomous virtual agents. Both the pursuer and the evader have limited amounts of energy, which is used up in movement, so they have to evolve to move economically. These time- series are then fed into a custom 3-D movie generator [14].

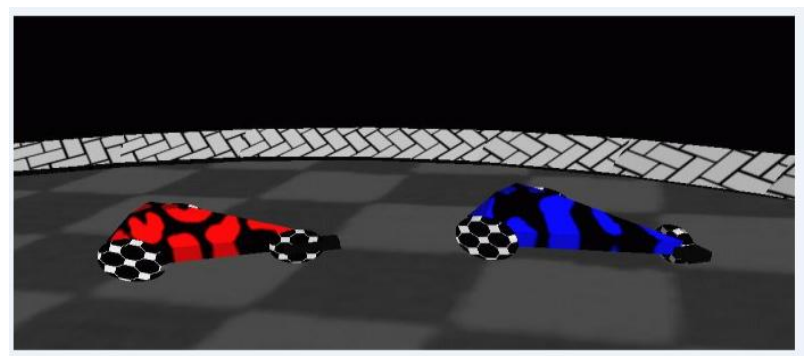

Fig 7 Movie sequences behavior generated by dynamical recurrent neural network controllers [15]

Brain Computer Interface based on Neural Networks Brain Computer Interface (BCI) is one of hopeful interface technologies between human and machine. Brain computer interface is also called mind machine interface (MMI).

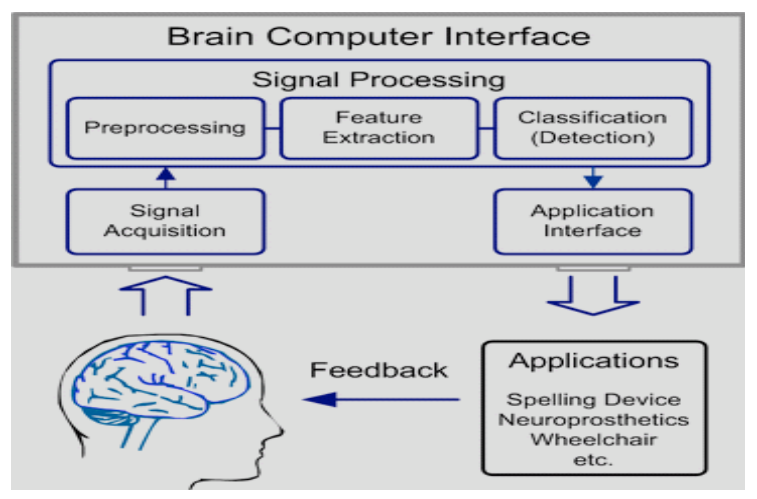

Fig 8 Schematic of a brain computer interface

Brain Computer Interface (BCI) is a communication channel connecting a brain to a computer or other electronic device that enables signals from the brain to direct interaction with external activity, such as control of a cursor or users can inputs phone numbers by gazing at the keypad of monitor. The interface enables a direct communication pathway between the brain and the object to be controlled [16]. Brain Computer Interface can enable a person suffering from paralysis to write a book or control a motorized wheelchair. Since the EEG 
was first described by Hans Berger in 1929 at present, EEG (electroen-cephalography) is the optimal choice for BCI implementation [17]. However, brain waves are very weak and there exist many kinds of noises. Therefore, what kinds of features are useful, how to extract the useful features, how to suppress noises, and so on are very important. So Neural networks can be applied for classification of noisy signals from sensitive signals and improve the accuracy of mental task classification [18]. Like other communication and control Systems, BCI's have inputs, outputs, and translation algorithms that convert the Former to the latter. BCI operation depends on the interaction of two adaptive controllers, the user's brain, which produces the input and the system itself, which translates that activity into output (i.e., specific commands that act on the external world) [19]. Over the past five years, the volume and pace of BCI research have grown rapidly. In 1995 there were no more than six active BCI research groups, now there are more than twenty [20][21].

Understanding and Description of Object Behaviors: This is one of the hot topics in computer vision. Trajectory analysis is one of the basic problems in behavior understanding. The learning of trajectory patterns can be used to detect anomalies and predict object trajectories. Trajectory analysis is the basis of scene understanding. The basic problem of behavior understanding is the target motion trajectory analysis. Currently the most common trajectory analysis methods focus on the geometric characteristics of the whole trajectory and neglect the semantic information related to the common sub-trajectories [22]. Here the task is all about the modeling or creating representation of object behaviors using detailed, learnt statistical models. A statistically based model of object trajectories is presented which is learnt from the observation of long image sequences. Trajectory data is supplied by a tracker using Active Shape Models, from which a model of the distribution of typical trajectories is, learnt [23]. The techniques being developed will allow models of characteristic object behaviors to be learnt from the continuous observation of long image sequences. It is seen that these models of characteristic behaviors representation will have a number of uses, particularly in automated surveillance and event recognition without the need for high-level scene/behavioral knowledge. In automatic method for learning model of semantic region by analyzing the trajectories of moving objects in the scene or frame should be done in following manners. First the trajectory is encoded to represent both the position of the object and its instantaneous velocity, and then hierarchical clustering algorithm is applied to cluster the trajectories according to different spatial and velocity distributions. In each cluster, trajectories are spatially close, have similar velocities of motion and represent one type of activity pattern. Based on the trajectory clusters, the statistical models of semantic region in the scene are generated by estimating the density and velocity distributions of each type of activity pattern [24].

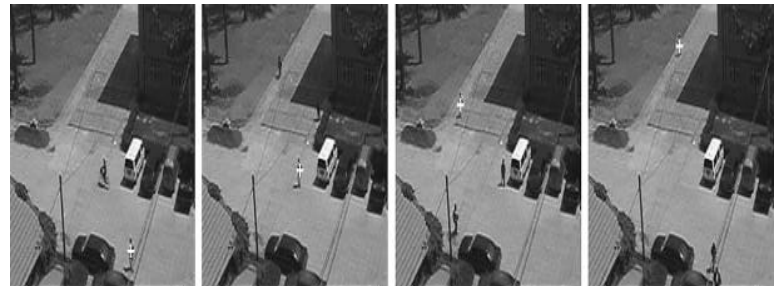

Fig 9 Tracking of pedestrian in a campus scene

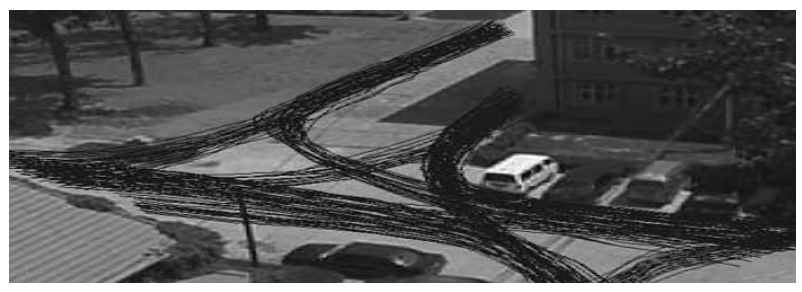

Fig 10 Trajectories in outdoor campus scene.

The model is learnt in an unsupervised manner by tracking objects over long image sequences, and is based on a combination of a neural network implementing Vector Quantization and a type of neuron with shortterm memory capabilities. The distribution patterns of trajectories are learnt and recognized using a hierarchical self-organizing neural network. Models of the trajectories of pedestrians have been generated and used to assess the typicality of new trajectories (allowing the identification of incidents of interest' within the scene), predict future object trajectories, and randomly generate new trajectories [25]

Artificial Neural Networks in Computer Graphics:

Now a days ANN's play an important role in graphics fields also. Graphics designers are trying to synthesis or merging actual or real images with computer generated images for enhancing visualization of the output image. Some of the most realistic images can be generated using Radiosity techniques. Radiosity for computer graphics was first introduced in [Goral et al.1984]. The idea was to simulate energy (light) transference from diffuse surfaces. The Radiosity method was further developed to account for the interaction of diffuse reflection between objects in scenes Radiosity for Virtual Reality Systems (ROVER) is emerging field for researchers to create virtual reality using ANN's [26].

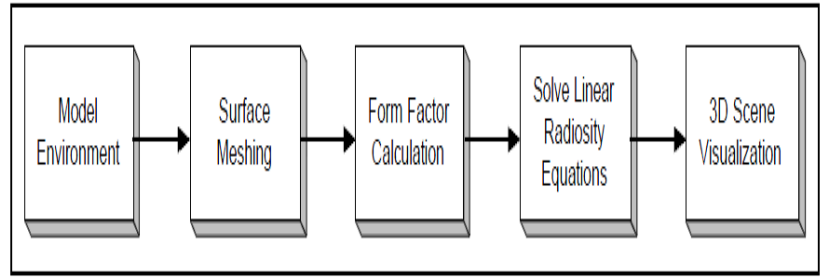

Fig 11 Graphics Pipeline for Radiosity

It is generally recognized that traditional implementation of Radiosity is computationally very expensive and therefore not feasible for use in VR (virtual Reality) systems where practical data sets are of huge complexity [27].There are two new methods and several hybrid techniques to the Radiosity research 
community on using Radiosity in VR applications. On the left column flyby, walkthrough and a virtual space are first introduced and on the left. On the right, one method using Neural Network technology is shown.

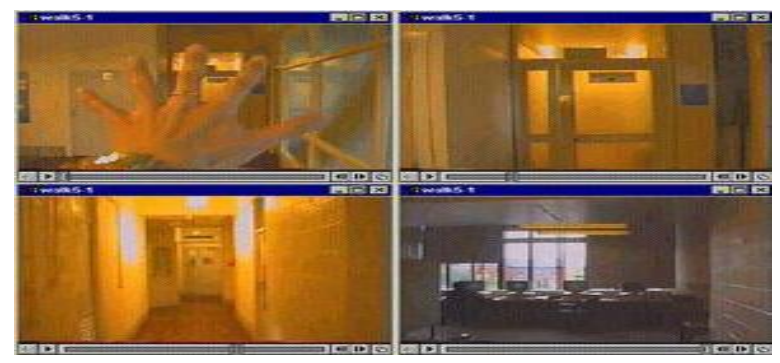

Fig 12 ROVER Learning Method Using ANN's [28]

\section{Autonomous walker \& Underwater Robot}

Autonomous walking robots based on the concepts of modularization. The problems involved with making an autonomous walker break into several groups of functionally. Trying to build a single grand model that fits your idea of a walker is difficult at best. It's fortunate that like almost all complex problems, it reduces to more manageable smaller problems [29]. The research in this area involves combining biology, mechanical engineering and information technology in order to develop the techniques necessary to build a dynamically stable legged vehicle controlled by a neural network. Underwater robots and underwater machines are counted on helping the salvage procedure, escaping prevention of pollution, lifesaving at sea, and scientific exploration in the ocean and so on. In the underwater robot, therefore, Autonomous Underwater Vehicles (AUVs) have been developed actively during recent year. However, AUVs have various problems which should be solved such as motion control, acquisition of sensors' information, behavioral decision self localization and so on. The SOM (self organizing maps) and Recurrent Neural Network (RNN) are used for this purpose [30].
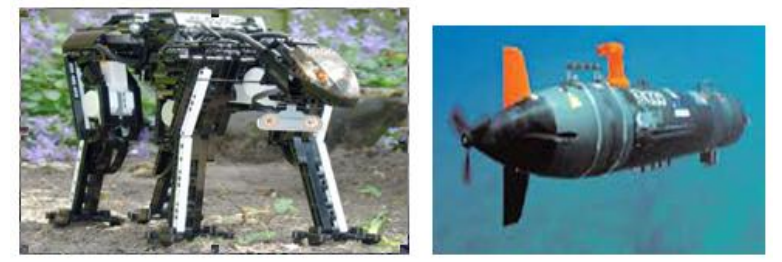

Fig 13 Autonomous walker \& under water Robot

Facial Animation: Modeling and animation of human faces is one of the most difficult tasks in computer graphics today, even more so when life is to be breathed into digitized versions of real, well-known individuals. Facial animations created using hierarchical B-spline as the underlying surface representation. Neural networks could be use for learning of each variation in the face expressions for animated sequences. Some clustering and machine learning methods are combined together to learn the correspondence between the speech acoustic and face animation parameters. The main learning machine used for speech facial animations are HMM, SVM and Neural Networks [31].

a) 2D Animation: Two-dimensional facial animation is commonly based upon the transformation of images, including both images from still photography and sequences of video. Image morphing is a technique which allows in-between transitional images to be generated between a pair of target still images or between frames from sequences of video.

b) 3D Animation: Three-dimensional head models provide the most powerful means of generating computer facial animation. One of the earliest works on computerized head models for graphics and animation was done by Parke. The model was a mesh of 3D points controlled by a set of conformation and expression parameters.

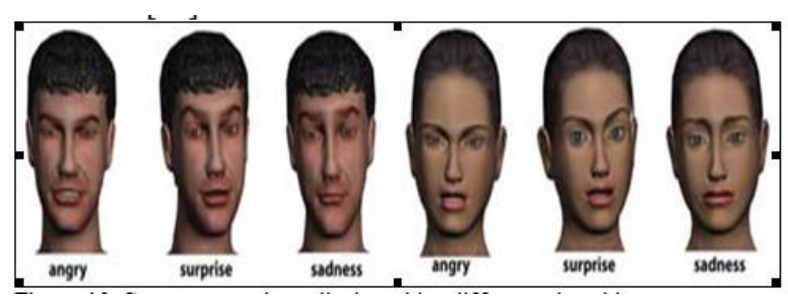

Fig 14: Some expressions displayed by different virtual humans.

Above figure shows different facial expression from different peoples in different situations. Thus neural networks and some others machine learning tools are used for recognisation of expression.

Neural Networks Strengthens Technology behind antivirus functioning: Artificial neural networks and artificial intelligence techniques have played increasingly important role in antivirus detection and providing enough strengths to the internal functioning of antivirus so it can detect and fix all kind of viruses. At present, some principal artificial intelligence techniques applied in antivirus detection including heuristic technique, artificial neural network, data mining, agent technique, artificial immune and it believes that it will improve the performance of antivirus detection systems. IBM's neural network boot detection technology provides additional security by mimicking human neurons in learning the difference between infected and uninfected boot records. By being shown many examples of viruses and non-viruses, the neural network learned to recognize viruses better than traditional heuristics hand-tuned by virus researchers. This neural network can detect an extremely high percentage of new and unknown boot record viruses automatically. Together, these technologies provide Norton Antivirus customers superior protection against both known and unknown boot sector viruses [32].

Speech Reading (Lip reading): Lip-Reading has been practiced over centuries for teaching deaf and dumb to speak and communicate effectively with the other People. Speech Vision, another term for lip-reading or speech reading, is a technique of understanding speech 
by visually interpreting the movements of lips, face and tongue using the information provided (if any) by the context, language, and any residual hearing. We convert the video of the subject speaking different words into images and then images are further selected manually for processing [33]

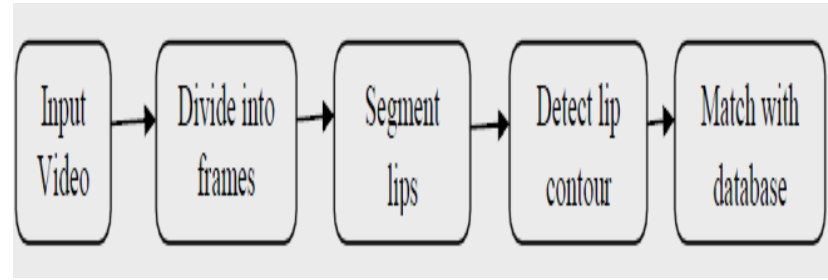

Fig 15 Block diagram for lip reading system [34]

The input to a lip-reading system consists of a video which is first divided into frames. Lips are then segmented on the first frame and then lip contour is determined. An object tracking mechanism is then used to track the motion of lips on subsequent frames. The position of lip contour on each frame is stored in a database. A separate database of characters is also maintained. The position of lip contour is matched with the characters to determine what the speaker has spoken [35].

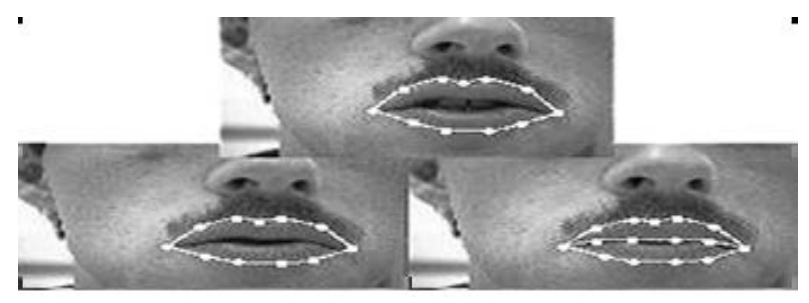

Fig 16 Lips reading with different mode of variation

\section{Applications of Artificial Intelligence}

Data Mining or Knowledge Extraction: Data mining is a fast-growing area. Data mining is a part of a process called KDD knowledge discovery in databases. This process consists basically of steps that are performed before carrying out data mining such as data selection, data cleaning, pre-processing of data, and data transformation [36]. "Data Mining is the use of computer algorithms to discover hidden patterns and unsuspected relationships among elements in a large data set. AI is a broader area than machine learning. AI systems are knowledge processing systems. Knowledge representation, knowledge acquisition, and inference including search and control, are three fundamental techniques in $\mathrm{AI}$

Knowledge representation: Data mining seeks to discover interesting patterns from large volumes of Data. These patterns can take various forms, such as association rules, classification rules, and decision trees, and therefore, knowledge representation becomes an issue of interest in data mining.
Knowledge acquisition: The discovery process shares various algorithms and methods with machine learning for the same purpose of knowledge acquisition from data learning from examples.

Knowledge inference: The patterns discovered from data need to be verified in various applications and so deduction of mining results is an essential technique in data mining applications. Therefore knowledge representation knowledge acquisition and knowledge inference, the three fundamental techniques in $\mathrm{AI}$ are all relevant to data mining [37].

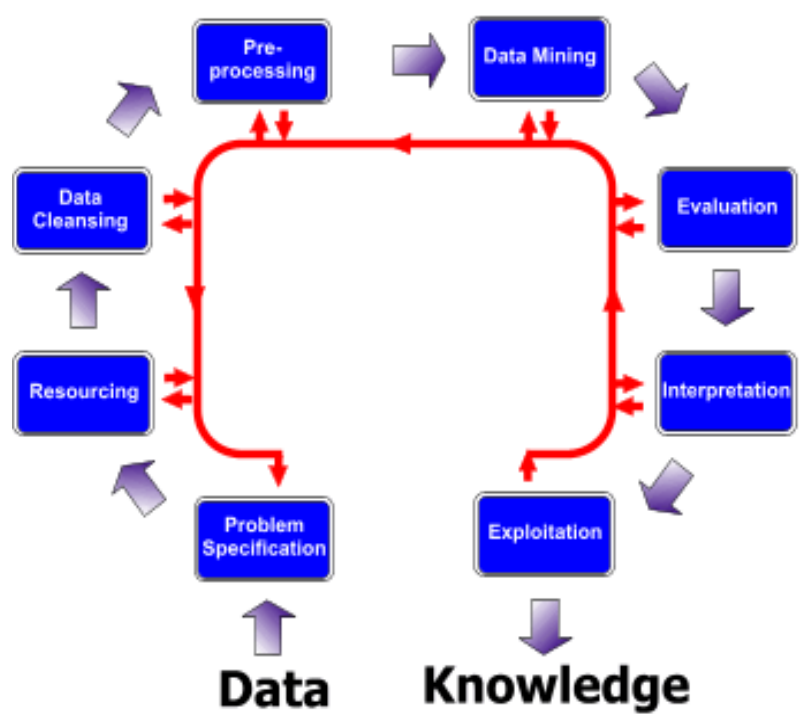

Fig 17 Data mining life cycle

Artificial Systems: The first expert system was developed in 1965 by Edward Feigenbaum and Joshua Lederberg of Stanford University in California called DENDRAL. Expert systems may be considered to be a subset of Artificial Intelligence. Expert system is an artificial intelligence program that has expert-level knowledge about a particular domain and knows how to use its knowledge to respond properly. Domain refers to the area within which the task is being performed. Ideally the expert systems should substitute a human expert. Expert systems are Artificial Intelligence programs that have expert level knowledge about a particular domain and know how to use knowledge to responds properly. Domain refers to the area within which a particular task is being performed. Ideally an Expert system should substitute a human expert. Edward Feigenbaum of Stanford University has defined expert system as "an intelligent computer program that uses knowledge and inference procedures to solve problems that are difficult enough to require significant human expertise for their solutions [38].

Every expert system consists of two principal parts:

A) The knowledge Base

B) Reasoning or Inference Engine 


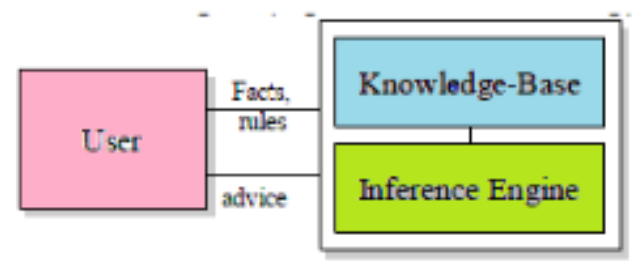

Fig18 Expert system using AI approach

Expert system $=$ Knowledge + inference Engine

Program in traditional computer = Data structure+ algorithm

Knowledge base: The knowledge base stores all the facts and rules about a particular problem domain. It makes these available to the inference engine in a form that it can use. The facts may be in the form of background information built into the system or facts that are input by the user during a consultation. The rules include both the production rules which applied to the domain of the expert system. The heuristics of rulesof-thumb that are provided by the domain expert in order to make the system find solutions more efficiently by taking short cuts.

Inference Engine: The inference engine is the program that locates the appropriate knowledge in the knowledge base, and infers new knowledge by applying logical processing and problem-solving strategies [39].

Soft Computing: Soft computing is a partnership of computing techniques. The partnership includes fuzzy logic models and particle swarm techniques. All these computing techniques have been recognized as attractive alternatives to the standard well established "hard computing" paradigms such as stochastic and statistical methods bound by the concept called NP complete [40].The dominant aim of soft computing is to exploit the tolerance for imprecision and uncertainty to achieve tractability, robustness and low solutions cost with less time then hard computing. The principal constituents of soft computing are fuzzy logic, neurocomputing, and probabilistic reasoning, with the latter subsuming genetic algorithms, belief networks, chaotic systems, and parts of learning theory. In the partnership of fuzzy logic, neurocomputing, and probabilistic reasoning, fuzzy logic is mainly concerned with imprecision and approximate reasoning; neurocomputing with learning and curve-fitting; and probabilistic reasoning with uncertainty and belief propagation [41].

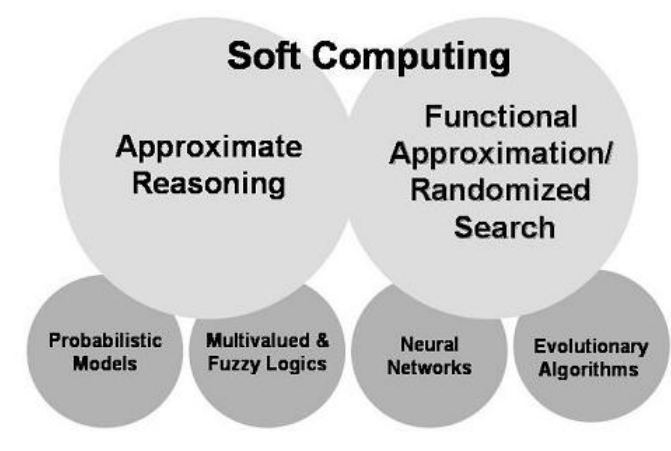

Fig 19 Soft computing a Hybrid Approach

The main components of Soft Computing are fuzzy logic neural networks, probabilistic reasoning, genetic algorithms and chaos theory shown in figure 18. In this framework it is shown that Fuzzy logic acts like kernel of the soft computing. Fuzzy logic main characteristic is the robustness of its interpolative reasoning mechanism. Within Soft Computing, Fuzzy Logic occupies a special place because it can be used as a springboard for generalization of any theory, including its partners in SC consortium. In Soft computing Fuzzy logic is mainly concerned with imprecision and approximate reasoning, neural networks with learning, Probabilistic reasoning with uncertainty and propagation of belief, Genetic algorithms with global optimization and search and Chaos theory with nonlinear dynamics. Each of these computational paradigms provides us with complementary reasoning and searching methods to solve complex, real-world problems. In large scope, FL, $\mathrm{NN}, \mathrm{PR}$, and GA are complementary rather that competitive [42][43][44].

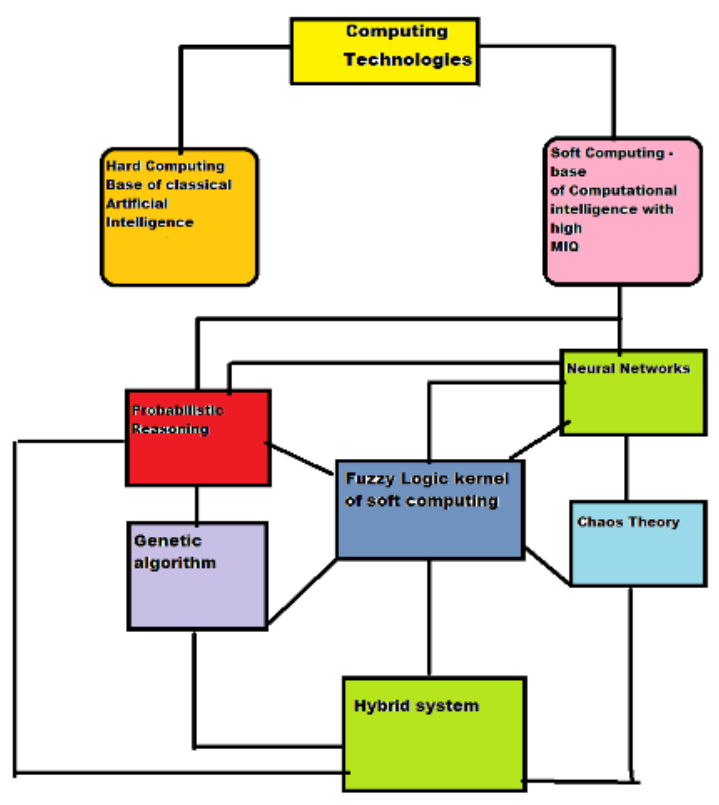

Figure 20 The main components of soft computing

Natural Language Processing: Natural Language Processing (NLP) is a subfield of Artificial Intelligence. NLP is a approach to analyzing text that is based on 
both a set of theories and a set of technologies. NLP is a form of human-to-computer interaction where the elements of human language, be it spoken or written, are formalized so that a computer can perform valueadding tasks based on that interaction. Natural Language Processing concerned with the interactions between computers and natural (human) languages like Hindi, English, and Spanish etc. The foundations of NLP lie in a number of disciplines, viz. computer and information sciences, linguistics, mathematics, electrical and electronic engineering, artificial intelligence and robotics, psychology, etc [45].

Goal: The goal of NLP as stated above is "to accomplish human-like language processing". The choice of the word 'processing' is very deliberate, and should not be replaced with Understanding.

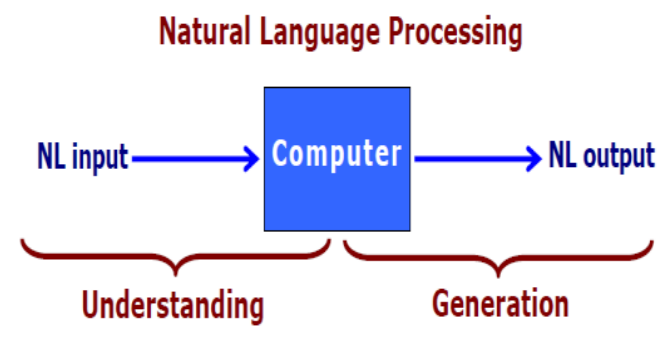

Fig 21 NLP Communication Model

Robotics: Robotics is one field within artificial intelligence. The term "artificial intelligence" is defined as systems that combine sophisticated hardware and software with elaborate databases and knowledge-based processing models to demonstrate characteristics of effective human decision making. It involves mechanical, usually computer-controlled, devices to perform tasks that require extreme precision or tedious or hazardous work by people. Traditional Robotics uses Artificial Intelligence planning techniques to program robot behaviors and works toward robots as technical devices that have to be developed and controlled by a human engineer. The Autonomous Robotics approach suggests that robots could develop and control themselves autonomously. These robots are able to adapt to both uncertain and incomplete information in constantly changing environments. It lets a simulated evolution process develop adaptive robots. The most intriguing use of robotics, however, is one that Mitsubishi just recently created. They have demonstrated robotic fish running AI programs, and swim around in the water. In fact, they look so real that only a close examination of the fish will reveal their robotic eyes [46]
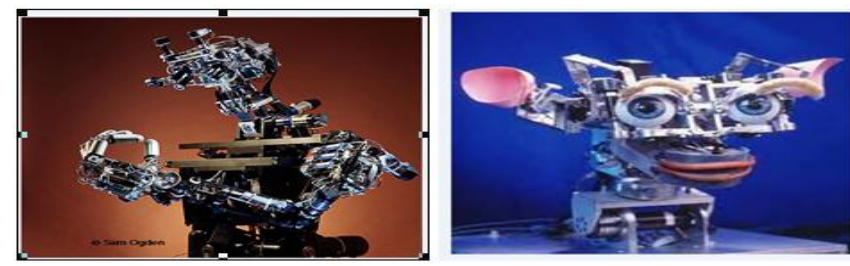

Fig $22 \mathrm{Cog}$, a humanoid robot from MIT \& Kismet robot advanced version of $\mathrm{Cog}$
Two of the many research projects of the MIT Artificial Intelligence department include an artificial humanoid called Cog and his baby brother Kismet. What the researchers learn while putting the robots together will be shared to speed up development. Once finished, Cog will have everything except legs, whereas Kismet has only a 3.6-kilogram head that can display a wide variety of emotions. Kismet is an autonomous robot designed for social interactions with humans and is part of the larger $\mathrm{Cog}$ Project. This project focuses not on robot-robot interactions, but rather on the construction of robots that engage in meaningful social exchanges with humans [47]

The GOALS of most robotic research projects is the advancement of abilities in one or more of the following technological areas Artificial intelligence, effectors and mobility, sensor detection and especially robotic vision, and control systems.

Swarm Intelligence: This is an approach to, as well as application of artificial intelligence similar to a neural network. Swarm Intelligence (SI) is an innovative distributed intelligent paradigm for solving optimization problems that originally took its inspiration from the biological examples by swarming, flocking and herding phenomena in vertebrates. The term SI has come to represent the idea that it is possible to control and manage complex Systems of interacting entities even though the interactions between and among the entities being controlled is, in some sense. Swarm Intelligence is the property of a system whereby collective behaviors of unsophisticated agents interacting locally with their environment cause coherent functional global patterns to emerge. Swarm Intelligence (SI) is a relatively new paradigm being applied in a host of research settings to improve the management and control of large numbers of interacting entities such as communication, computer and sensor networks, satellite constellations and more. The underlying features of SI are based on observations of social insects. Ant colonies and beehives, for example, have the interesting property that large numbers of them seem to conduct their affairs in a very organized way with seemingly purposeful behavior that enhances their collective survival [48],[49].

\section{Swarm Intelligence-based Applications:}

- Complex interactive virtual environments generation in movie industries'.

- Cargo arrangement in Airline companies.

- $\quad$ Route scheduling in delivery companies

- $\quad$ Routing packets in telecommunication networks.

- $\quad$ Power grid optimization control

- Data Clustering, Data routing in Sensor Network

- Unmanned vehicles controlling in the U.S. military's

- $\quad$ Planetary mapping and micro-satellite controlling in NASA. 
Artificial Intelligence in Aviation Industry: Airlines use expert systems in planes to monitor atmospheric conditions and system status. The plane can be put on autopilot once a course is set for the destination [50]. Recently researchers proposed model of aircraft intelligent landing system with dynamic neuro-fuzzy controller that enhances the fault tolerant capabilities of a high performance civil aviation aircraft during the landing phase when subjected to severe winds and failures such as stuck control surfaces [51].

Artificial Intelligence in Games: Modern computer games usually employ 3D animated graphics (and recently also $3 \mathrm{D}$ sound effects) to give the impression of reality. The AI found in most computer games is no AI (in the academic sense), but rather a mixture of techniques which are although related to AI mainly concerned with creating a believable illusion of intelligence [52]. The phrase "game AI" covers a diverse collection of programming and design practices including path finding, neural-networks, and models of emotion and social situations, finite state machines, rule systems, decision-tree learning, and many other techniques.

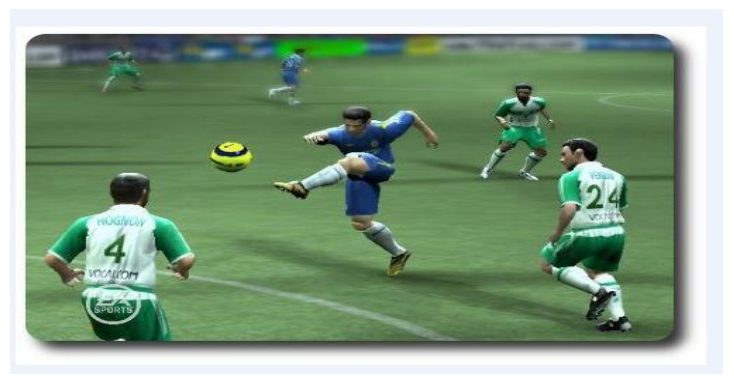

Fig 23 AI Bots in a video game

Ai Also Used In : Pattern recognition, Optical character recognition, Handwriting recognition, Speech recognition, Face recognition, Artificial Creativity, Computer vision, Virtual reality and Image processing, Diagnosis (artificial intelligence), Game theory and Strategic planning, Translation and Chatter bots, Automated Car Steering, Cheque fraud protection etc [53].

\section{Conclusion}

The computing world has a lot to gain or benefits from neural networks approaches. Their ability to learn by example makes them very flexible and powerful. Furthermore there is no need to devise an algorithm in order to perform a specific task i.e. there is no need to understand the internal mechanisms of that task. They are also very well suited for real time systems because of their fast response and computational times which are due to their parallel architecture. The goal of artificial intelligence is to create computers whose intelligence equals or surpasses humans'. Achieving this goal is the famous "AI problem from last decade researchers are trying to close the gap between human intelligence and artificial intelligence. This Review Paper explained important applications of artificial intelligence \& neural networks to create intelligent behavior. Artificial intelligence in the future will try to make machines and computers much more sophisticated than the ones that we have today. It is expected that in the future, such machines will be developed, which have basic common sense, similar to human beings, although pertaining to specific areas only. It is also expected that the human mind functions, such as learning by experience, learning by rehearsal, cognition and perception will also be performed by future intelligent machines. Examples were given to show how AI and ANN's are used in applications like Brain computer interface, Expert system, Swarm intelligence, and soft computing.

\section{The Future}

The future of Neural Networks is wide open, and may lead to many answers and/or questions. Some of the questions on which researchers are currently working are.

a) Will a machine ever be conscious of its own existence?

b) What does it mean to be human?

c) Will neural networks ever fully simulate the human brain?

d) Will they be as complex and as functional?

\section{References}

[1] George F Ludger "Artificial Intelligence Structures and strategies for complex problem solving" 5th Edition, Pearson, 2009.

[2] Girish Kumar jha, "Artificial Neural Networks and its applications" international journal of computer science and issues 2005.

[3] Nils J Nilsson American Association for Artificial Intelligence" AI magazine 2005.

[4] http://www.differencebetween.com/differencebetween-strong-ai-and-vs-weak-ai/

[5] Tyler Cowen and Michelle Dawson "What does the Turing test really mean? And how many human beings (including Turing) could pass? George Mason University Department of Economics, and University of Montreal, July 3. 2009.

[6] Ajith Abraham." Artificial Neural Networks" Oklahoma State University, Stillwater OK, USA 2006

[7] Zhi-Hua Zhou, "Rule Extraction Using Neural Networks or For Neural Networks" National Laboratory for Novel Software Technology, Nanjing University, Nanjing 210093, China 2007

[8] Fiona Nielsen, "Neural Networks algorithms and applications" Neil's Brock Business College, Dec 2001.

[9] Jacek M. Zurada, "Introduction to Artificial Neural System", Jaico publishing house 2006

[10] Fausett, L. "Fundamentals of Neural Networks" Prentice Hall, USA. 1996 
[11] Sutton, R. S. and Barto, A. G. "Reinforcement Learning: An Introduction Cambridge, MA MIT Press 1999

[12] Saumya Bajpai, Kreeti Jain, and Neeti Jain.,"Artificial Neural Networks" International Journal of Soft Computing and Engineering (IJSCE) ISSN: 2231-2307, Volume-1, Issue-NCAI2011, June 2011

[13] Seven G Ficici and Jordan B. Pollock. "Statistical Reasoning Strategies in the pursuit and Evasion domains. Demo Lab, Brandeis University, USA 2001.

[14] Jyoti Singh and Pritee Gupta. "Advance Applications of Artificial Intelligence and Neural Networks" Akgec Journal of Technology, vol.1, no.2, in 2010.

[15] http://www.demo.cs.brandeis.edu/pr/neural_control lers/evo_control.html.

[16] Ming Cheng, Xiaorong GAO, Shangkai GAO, Senior Member, IEEE, and Dingfeng Xu "Design and Implementation of a Brain-Computer Interface With High Transfer Rates" 2002.

[17] H. Berger, "Uber das Electrenkephalogramm des Menchen," Arch Psychiat Nervenkr, vol. 87, pp. 527-570, 1929.

[18] Jonathan R. et al "Brain-Computer Interface Technology" A Review of the First International Meeting" IEEE transactions on Rehabilitation Engineering, vol. 8, no. 2, June 2000.

[19] J. R. Wolpaw et al., "Brain-computer interface technology: A review of the first international meeting," IEEE Trans. Rehab. Eng., vol. 8, pp. 164-173, June 2000.

[20] Wolpaw, J.R. "Brain-computer interfaces signals, methods, and goals Neural Engineering" Conference Proceedings. First International IEEE EMBS Conference 2003.

[21] Kenji Nakayama Kiyoto Inagaki, "A Brain Computer Interface Based on Neural Network with Efficient Pre-Processing" Graduate School of Natural Science and Technology, Kanazawa Univ. Kakuma-machi, Kanazawa, 920-1192, Japan 2007.

[22] Yuanfeng Yang et al. "Trajectory Analysis Using Spectral Clustering and Sequence Pattern Mining" Journal of Computational Information Systems 2012.

[23] Neil Johnson, David Hogg "Learning the distribution of objecttrajectories for eventrecognition"School of Computer Studies, The University of Leeds, Leeds LS2 9JT, UK 1999.

[24] Lei Gao et al. "Automatic Learning of Semantic Region Models for Event Recognition" School of Computer. Sci. \& Technol., Beihang Univ., Beijing 2009

[25] Weiming $\mathrm{Hu}$, Dan Xie, and Tieniu Tan, Senior Member, IEEE "A Hierarchical Self-Organizing Approach for Learning the Patterns of Motion Trajectories" IEEE transactions on neural networks, vol.15, no. 1, January 2004.
[26] Brennan J. Rusnell. "Radiosity for Computer Graphics" University of Saskatchewan 2007.

[27] Brennan J. Rusnell, "Radiosity for Computer Graphics" University of Saskatchewan 2007.

[28] http://tralvex.com/pub/nap/\#Radiosity for Virtual Reality Systems (ROVER).

[29] Takemura Y and Ishii K; "Dynamics classification of underwater robot and Introduction to controller adaptation" Dept. of Mech. \& Electron. Eng, Nippon Bunri Univ., Oita, Japan dec 2010

[30] Tamara Knutsen et al, "Designing an Underwater Eel-Like Robot and Developing Anguilliform Locomotion Control" Harvard University 2004

[31] S.Garchery, A. Egges, N. Magnenat-Thalman. "Fast Facial Animation Design for Emotional Virtual Humans" MIRALab, University of Geneva, Geneva, Switzerland 2007

[32] Junaid Akhtar and Ayesha Farrukh "Applications of Artificial Neural Networks" Research trends in artificail intelligence 2004.

[33] Abhay Bagai et al "Lip-Reading using Neural Networks "IJCSNS International Journal of Computer Science and Network Security, vol.9 No.4, April 2009.

[34] Harshit Mehrotra and Prof. M.C. Srivastava. "Lip reading" Department of electronics and communication engineering jaypee institute of information technology university noida may 2009.

[35] T. Chen, "Audiovisual speech processing lip reading and lip synchronization," IEEE Signal Processing Mag., vol. 18, pp. 9 21, January 2001.

[36] Hebah H. O. Nasereddin "Stream Data Mining" Department of computer Information system Faculty of IT Amman Arab University for Graduate Studies Amman Jordan 2009.

[37] Xindong Wu, Senior Member, IEEE "Data Mining: An AI Perspective" vol.4 no 2 (2004)

[38] Satvika Khanna et al. "Expert Systems Advances in Education" NCCI 2010 -National Conference on Computational Instrumentation CSIO Chandigarh, INDIA, 19-20 March 2010

[39] O. N. Garcia, R. A. Perez, et. al, "On Teaching AI and Expert Systems Courses", IEEE Transactions on Education, Vol. 36, No.1 Feb (1993).

[40] Yanbo Huang. "Advances in Artificial Neural Networks - Methodological Development and Application" United States Mississippi 38776, USA;

[41] http://modo.ugr.es/en/soft_computing

[42] Zadeh L.A. The roles of fuzzy logic and soft computing in the conception, design and deployment of intelligent systems. BT Technol J. 14(4): 32-36, 1994

[43] Aliev R.A. and Aliev R.R., Soft Computing, volumes I, II, III. Baku: ASOA Press, 1997-1998 (in Russian).

[44] Aliev R., Bonfig K., and Aliew F., Soft Computing. Berlin: Verlag Technic, 2000.

[45] Gobinda G. Chaudhary "Natural Language Processing" Dept. of Computer and Information 
Sciences University of Strathclyde, Glasgow G1 1XH, UK 2003

[46] Robert Chin (Qin) Mrs. Baron World Lit. Honor. "Artificial Intelligence A Window to Mankind Feb.1999

[47] http://www.electronicsteacher.com/robotics/current -research.php

[48] Mark Fleischer "Foundations of Swarm Intelligence From Principles to Practice" Institute for Systems Research University of Maryland College Park, Maryland 2005

[49] W. Poundstone, "The Recursive Universe: Cosmic Complexity and the Limits of Scientific Knowledge". Chicago, Ill Contemporary Books, 1985

[50] P Venketesh, R Venkatesan, "A Survey on Applications of Neural Networks and Evolutionary Techniques in Web Caching", IETE Tech Rev 2009.

[51] Kaijun Xu.” Dynamic neuro-fuzzy control design for civil aviation aircraft in intelligent landing system. Dept. of Air Navig. Civil Aviation Flight Univ. of China 2011.

[52] Eike.F Anderson.,'Playing smart artificial intelligence in computer games" The National Centre for Computer Animation (NCCA) Bournemouth University UK.

[53] K.R. Chaudhary "Goals, Roots and Sub-fields ofArtificial Intelligence. MBM Engineering College, Jodhpur, India 2012

\section{Short Bio Data for the Author's}

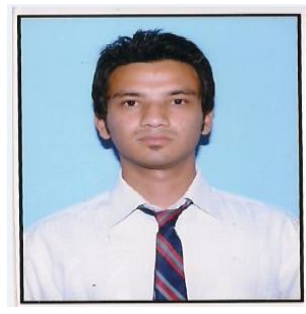
research interests lies in Computer Networks, Grid Computing, Artificial Neural Networks and soft computing.

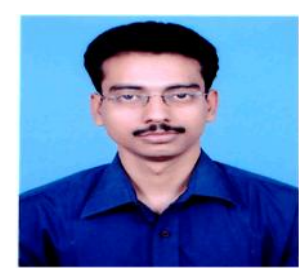

Mr.Gour Sundar Mitra Thakur currently he is working as an Asst. Professor (CSE/IT) Lovely Professional University, Punjab, India. B.Tech (C.S.E), M.Tech (C.S) and Pursuing Ph.D from National Institute of Technology, Durgapur in Mathematics. His Areas of Interests are Fuzzy Logic and Fuzzy Mathematics, Soft Computing, Intelligent Systems and Neural Networks. 